\section{Mitochondrion-Toxic Compounds Need to be avoided in Leigh Syndrome}

Sir,

We read with interest the article by Huda et al. about the anesthetic management of a 6-year female with Leigh syndrome, who underwent surgery for strabismus. ${ }^{1}$ The anesthetic management included propofol and remifentanil intravenously for induction and maintenance of anesthesia. ${ }^{1}$ It was concluded that depolarising muscle relaxants may trigger rhabdomyolysis, while application of non-depolarising muscle relaxants may be complicated by prolonged recovery time. ${ }^{1}$ We have the following comments and concerns.

The main shortcoming of the study is that the diagnosis of Leigh syndrome was not genetically confirmed. ${ }^{1}$ Knowing the genetic background of Leigh syndrome is not only crucial for predicting the outcome, but also for genetic counselling. Genetic counselling additionally requires confirmation of the mutation in first-degree relatives, and documentation of the trait of inheritance. At least we should be informed about the clinical presentation of other first-degree relatives, in particular the mother and father, as the majority of the patients with Leigh syndrome carry a mutation in a nuclear mitochondrial gene. Mutations in $>75$ genes have been so far identified. ${ }^{2}$

A second shortcoming of the study is that no results on biochemical investigations of the muscle homogenate were presented. Since the patient obviously had myopathy of the extra-ocular muscles resulting in strabismus/ophthalmoparesis, it is crucial to know if other muscles were clinically or biochemically affected, as well. Missing in this respect are the results of the clinical neurologic investigation. Particularly, we should know if muscle groups such as the axial muscles, bulbar muscles, respiratory muscles, or the limb muscles were additionally affected.

Concerning the anesthetic management, we should know if the post-anesthetic recovery time was prolonged, if muscle weakness increased after anesthesia, or if any signs of malignant hyperthermia-like symptoms developed during anesthesia. We also should know if serum lactate increased during anesthesia. For propofol, it is well-known that it is mitochondrion-toxic and may cause propofol-infusion syndrome. ${ }^{3}$ We should know if there were any manifestations of propofol-infusion syndrome in the indexpatient.

Overall, this interesting study has several limitations, which should be addressed before drawing final conclusions. Particularly, the diagnosis of Leigh syndrome should be genetically confirmed, the muscles of the index patient should be biochemically investigated, and first-degree relatives should be clinically and genetically investigated. Since intravenous propofol may be complicated by propofol-infusion syndrome in patients with a mitochondrial disorder, this compound should be given with caution in these patients.

\section{ETHICALAPPROVAL:}

The study was approved by the Institutional Review Board.

\section{CONFLICT OF INTEREST:}

The author declared no conflict of interest.

\section{AUTHOR'S CONTRIBUTION:}

JF: Design, literature search, discussion, first draft, critical comments.

\section{REFERENCES}

1. Huda AU, Yasir M. Anaesthesia management of a 6-year child with leigh syndrome undergoing strabismus surgery. J Coll Physicians Surg Pak 2021; 31(2):243. doi: 10.29271/ jcpsp.2021.02.243.

2. Baldo MS, Vilarinho L. Molecular basis of Leigh syndrome: $A$ current look. Orphanet J Rare Dis 2020; 15(1):31. doi: 10.1186/s13023-020-1297-9.

3. Finsterer J, Frank M. Propofol is mitochondrion-toxic and may unmask a mitochondrial disorder. J Child Neurol 2016; 31(13):1489-94. doi: 10.1177/0883073816661458.

Josef Finsterer

Department of Neurology, Klinik Landstrasse, Messerli Institute, Vienna, Austria

Correspondence to: Dr. Josef Finsterer, Department of Neurology, Klinik Landstrasse, Messerli Institute, Vienna, Austria

E-mail: fipaps@yahoo.de

Received: March 03, 2021; Revised: March 12, 2021;

Accepted: January 07, 2022

DOI: https://doi.org/10.29271/jcpsp.2022.02.270

\section{AUTHOR'S REPLY}

Sir,

First of all, we are thankful for the comments made by our respected readers. As we all know, case reports have several limitations in terms of drawing conclusion. Although, we can have some idea about the treatment and management of certain rare diseases, but as we mentioned that the scientific literature about anaesthesia management of patients with Leigh syndrome is very limited. It is difficult to include every detail of a case in a 'Letter to Editor' with limitations in word count.

Regarding our case, the genetic team of our hospital were involved in the diagnosis of this patient. All relevant genetic investigations were done and also genetic counselling was offered. Our patient had hypotonia of extra-ocular muscles 
along with facial dysmorphia and hypotonia of upper limb muscles.

Regarding the use of propofol in this patient, we would like to mention that there are two ways of anaesthesia induction and maintenance i.e., intravenous and inhalational agents. We know that patients with mitochondrial diseases undergoing anaesthesia with inhalational agents are also at risk of malignant hyperthermia $(\mathrm{MH})$, which is a life-threatening condition. In scientific literature, propofol-infusion syndrome is reported mainly in critically ill patients in intensive care unit (ICU). One recent literature review demonstrated a linear relationship between the cumulative dose of propofol; and both the number of features of propofol-infusion syndrome and the number of organ systems involved in adults. No such association was seen in children, and this may be related to the small number of published reports. ${ }^{1}$ The usual duration of this surgery varies between 60 to 90 minutes and; therefore, the risk of propofol-infusion syndrome in these patients is reduced. Additionally, this patient received anaesthesia before, as well; and propofol was used in that surgery without any complication. So, based on comparing the risks associated with both methods, we preferred the intravenous agent 'propofol'.

As we mentioned in our case report, the patient remained stable intraoperatively and no signs of $\mathrm{MH}$ were reported during and after surgery. Our patient recovered from anaesthesia very well. Her muscle power was same as before anaesthesia. There was no sign of respiratory distress in the recovery room. Based on our clinical assessment and also advice from the paediatrician, we did not need to do any further investigations. We kept her in the recovery room for a relatively longer period for better monitoring. Later, we transferred her to the ward, where she stayed for one day. There was no sign of propofol-infusion syndrome or worsening of muscle weakness in the ward.

\section{REFERENCE}

1. Hemphill S, McMenamin L, Bellamy MC, Hopkins PM. Propofol infusion syndrome: A structured literature review and analysis of published case reports. Br J Anaesth 2019; 122(4):448-59. doi: 10.1016/j.bja.2018.12.025.

Anwar-ul-Huda

Department of Anaesthesia, Security Forces Hospital, Riyadh, Kingdom of Saudi Arabia

Correspondence to: Dr. Anwar-ul-Huda, Department of Anaesthesia, Security Forces Hospital, Riyadh, Kingdom of Saudi Arabia

E-mail: hudaanwar90@yahoo.com 